\title{
EXISTENCE AND UNIQUENESS RESULTS FOR A COUPLED SYSTEM OF HADAMARD FRACTIONAL DIFFERENTIAL EQUATIONS WITH MULTI-POINT BOUNDARY CONDITIONS
}

\author{
Mohamed Houas and Khellaf Ould Melha
}

\begin{abstract}
(C) by University of Niš, Serbia | Creative Commons Licence: CC BY-NC-ND Abstract. In this paper, we have studied existence and uniqueness of solutions for a coupled system of multi-point boundary value problems for Hadamard fractional differential equations. By applying principle contraction and Shaefer's fixed point theorem new existence results have been obtained.
\end{abstract}

Keywords: multi-point boundary value problems; Hadamard fractional differential equations; Shaefer's fixed point theorem.

\section{Introduction}

Differential equations of fractional order have proved to be very useful in the study of models of many phenomenons in various fields of science and engineering, such as: electrochemistry, physics, chemistry, viscoelasticity, control, image and signal processing. For more details, we refer the reader to $[3,5,6,7,11,12,13,14,16$, 18]. There has been a sign cant progress in the investigation of these equations in recent years, see $[3,8,17,18,19]$. More recently, a basic theory for the initial boundary value problems of fractional differential equations has been discussed in $[1,3,14,16,20,22]$. On the other hand, existence and uniqueness of solutions to boundary value problems for fractional differential equations has attracted the attention of many authors, see for example, $[16,17,19]$ and the references therein. Moreover, the study of coupled systems of fractional order is also important in various problems of applied nature $[2,9,10,15,24,25]$. Recently, many people have established the existence and uniqueness for solutions of some fractional systems, see $[9,10,21,23,25]$ and the reference therein. In the last few decades, much attention has been focused on the study of the existence and uniqueness of solutions for boundary value problems of Riemann-Liouville type or Caputo type fractional

Received October 18, 2019; accepted February 20, 2020

2010 Mathematics Subject Classification. Primary 34A34; Secondary 34B10. 
differential equations, see $[21,23,24,25]$. There are few papers devoted to the research of the Hadamard fractional differential equations; see [2].

In this paper, we study the existence of solutions for a Hadamard coupled system of nonlinear fractional integro-differential equations given by:

$$
\left\{\begin{array}{l}
D^{\alpha} x(t)=f_{1}\left(t, y(t), D^{\delta} y(t)\right), 1<\alpha \leq 2, t \in[1, T], \\
D^{\beta} y(t)=f_{2}\left(t, x(t), D^{\sigma} x(t)\right), 1<\beta \leq 2, t \in[1, T], \\
x(1)=0, x(T)-\sum_{i=1}^{m} \lambda_{i} I^{p} x\left(\eta_{i}\right)=0, \\
y(1)=0, y(T)-\sum_{i=1}^{m} \mu_{i} I^{q} x\left(\xi_{i}\right)=0,
\end{array}\right.
$$

where $\sigma \leq \alpha-1, \delta \leq \beta-1 ; p, q>0 ; 1<\eta_{i}, \xi_{i}<T$ and $D^{\alpha}, D^{\beta}, D^{\delta}$ and $D^{\sigma}$ are the Hadamard fractional derivatives, $I^{p}$ and $I^{q}$ are the Hadamard fractional integrals and $f_{1}, f_{2}$ are continuous functions on $[1, T] \times \mathbb{R}^{2}$.

The rest of this paper is organized as follows. In section 2, we present some preliminaries and lemmas. Section 3 is devoted to existence of solution of problem (1.1). In section 4 an examples are treated illustrating our results.

\section{Preliminaries}

This section is devoted to the basic concepts of Hadamard type fractional calculus will be used throughout this paper [13].

Definition 2.1. The fractional derivative of $f:[1, \infty[\rightarrow \mathbb{R}$ in the sense of Hadamard is defined as:

$$
D^{\alpha} f(t)=\frac{1}{\Gamma(n-\alpha)}\left(t \frac{d}{d t}\right)^{n} \int_{1}^{t}\left(\log \frac{t}{s}\right)^{n-\alpha-1} \frac{f(s)}{s} d s, n-1<\alpha<n,
$$

where $n=[\alpha]+1,[\alpha]$ denotes the integer part of the real number $\alpha$ and $\log (t)=$ $\log _{e}(t)$.

Definition 2.2. The Hadamard fractional integral operator of order $\alpha>0$, for a continuous function $f$ on $[1, \infty[$ is defined as:

$$
I^{\alpha} f(t)=\frac{1}{\Gamma(\alpha)} \int_{1}^{t}\left(\log \frac{t}{s}\right)^{\alpha-1} \frac{f(s)}{s} d s, \alpha>0,
$$

where $\Gamma(\alpha):=\int_{0}^{\infty} e^{-u} u^{\alpha-1} d u$. 
Existence and uniqueness results for a coupled system of Hadamard fractional... 845

Lemma 2.1. Let $\alpha>0$. Then

$$
I^{\alpha} D^{\alpha} x(t)=x(t)+\sum_{i=1}^{n} c_{i}(\log t)^{\alpha-i}
$$

where $c_{i} \in \mathbb{R}, i=0,1,2, \ldots, n, n=[\alpha]+1$.

We give also an auxiliary lemma to define the solutions for the problem (1.1).

Lemma 2.2. Let $g \in C([1, T], \mathbb{R})$, the solution of the boundary value problem

$$
\left\{\begin{array}{l}
D^{\alpha} x(t)=g(t), 1<\alpha \leq 2, t \in[1, T] \\
x(1)=0, x(T)=\sum_{i=1}^{m} \lambda_{i} I^{p} x\left(\eta_{i}\right)
\end{array}\right.
$$

is given by:

$$
\begin{gathered}
x(t)=\frac{1}{\Gamma(\alpha)} \int_{1}^{t}\left(\log \frac{t}{s}\right)^{\alpha-1} \frac{g(s)}{s} d s \\
+\frac{(\log t)^{\alpha-1}}{\Pi}\left[\frac{\sum_{i=1}^{m} \lambda_{i}}{\Gamma(\alpha+p)} \int_{1}^{\eta_{i}}\left(\log \frac{\eta_{i}}{s}\right)^{\alpha+p-1} \frac{g(s)}{s} d s\right. \\
\left.-\frac{1}{\Gamma(\alpha)} \int_{1}^{T}\left(\log \frac{T}{s}\right)^{\alpha-1} \frac{g(s)}{s} d s\right]
\end{gathered}
$$

where

$$
\Pi=\frac{1}{(\log T)^{\alpha-1}-\frac{\Gamma(\alpha)}{\Gamma(p+\alpha)} \sum_{i=1}^{m} \lambda_{i}\left(\log \eta_{i}\right)^{p+\alpha-1}} .
$$

Proof. As argued in [13], for $c_{i} \in \mathbb{R}, i=1,2$, and by lemma 3, the general solution of equation of problem (2.4) is given by

$$
x(t)=\frac{1}{\Gamma(\alpha)} \int_{1}^{t}\left(\log \frac{t}{s}\right)^{\alpha-1} \frac{g(s)}{s} d s+c_{1}(\log t)^{\alpha-1}+c_{2}(\log t)^{\alpha-2} .
$$

Using the boundary conditions for (2.4), we find that $c_{2}=0$.

For $c_{1}$, we have

$$
\begin{gathered}
\frac{1}{\Gamma(\alpha)} \int_{1}^{T}\left(\log \frac{T}{s}\right)^{\alpha-1} \frac{g(s)}{s} d s+c_{1}(\log T)^{\alpha-1} \\
=\frac{\sum_{i=1}^{m} \lambda_{i}}{\Gamma(\alpha+p)} \int_{1}^{\eta_{i}}\left(\log \frac{\eta_{i}}{s}\right)^{\alpha+p-1} \frac{g(s)}{s} d s+\frac{c_{1} \Gamma(\alpha)}{\Gamma(p+\alpha)} \sum_{i=1}^{m} \lambda_{i}\left(\log \eta_{i}\right)^{p+\alpha-1} .
\end{gathered}
$$

which gives

$$
\begin{aligned}
c_{1}= & \frac{\frac{\sum_{i=1}^{m} \lambda_{i}}{\Gamma(\alpha+p)} \int_{1}^{\eta_{i}}\left(\log \frac{\eta_{i}}{s}\right)^{\alpha+p-1} \frac{g(s)}{s} d s}{(\log T)^{\alpha-1}-\frac{\Gamma(\alpha)}{\Gamma(p+\alpha)} \sum_{i=1}^{m} \lambda_{i}\left(\log \eta_{i}\right)^{p+\alpha-1}} \\
& -\frac{\frac{1}{\Gamma(\alpha)} \int_{1}^{T}\left(\log \frac{T}{s}\right)^{\alpha-1} \frac{g(s)}{s} d s}{(\log T)^{\alpha-1}-\frac{\Gamma(\alpha)}{\Gamma(p+\alpha)} \sum_{i=1}^{m} \lambda_{i}\left(\log \eta_{i}\right)^{p+\alpha-1}}
\end{aligned}
$$

Substituting the value of $c_{1}$ and $c_{2}$ in (2.7), we get (2.5). 


\section{Main Results}

Let us introduce the spaces $X=\left\{x: x \in C^{1}([1, T]), D^{\sigma} x \in C([1, T])\right\}$ and

$$
Y=\left\{y: y \in C^{1}([1, T]), D^{\delta} y \in C([1, T])\right\}
$$

endowed with the norm $\|x\|_{X}=\|x\|+\left\|D^{\sigma} x\right\|$; with

$$
\|x\|=\sup _{t \in[1, T]}|x(t)|,\left\|D^{\sigma} x\right\|=\sup _{t \in[1, T]}\left|D^{\sigma} x(t)\right|,
$$

and $\|y\|_{Y}=\|y\|+\left\|D^{\delta} y\right\| ;$ with

$$
\|y\|=\sup _{t \in[1, T]}|y(t)|,\left\|D^{\delta} y\right\|=\sup _{t \in[1, T]}\left|D^{\delta} y(t)\right| .
$$

Obviously, $\left(X,\|\cdot\|_{X}\right)$ and $\left(Y,\|\cdot\|_{Y}\right)$ are a Banach spaces. The product space $\left(X \times Y,\|(x, y)\|_{X \times Y}\right)$ is also Banach space with norm $\|(x, y)\|_{X \times Y}=\|x\|_{X}+\|y\|_{Y}$. Let us now introduce the quantities:

$$
\begin{aligned}
& N_{1}=\frac{(\log T)^{\alpha}}{\Gamma(\alpha+1)}+\frac{(\log T)^{\alpha-1}}{|\Lambda|}\left(\frac{\sum_{i=1}^{m} \lambda_{i}\left(\log \eta_{i}\right)^{p+\alpha}}{\Gamma(p+\alpha+1)}+\frac{(\log T)^{\alpha}}{\Gamma(\alpha+1)}\right), \\
& N_{2}=\frac{(\log T)^{\alpha-\sigma}}{\Gamma(\alpha-\sigma+1)}+\frac{\Gamma(\alpha)(\log T)^{\alpha-\sigma-1}}{\Gamma(\alpha-\sigma)|\Lambda|}\left(\frac{\sum_{i=1}^{m} \lambda_{i}\left(\log \eta_{i}\right)^{\alpha+p}}{\Gamma(\alpha+p+1)}+\frac{(\log T)^{\alpha}}{\Gamma(\alpha+1)}\right), \\
& N_{3}=\frac{(\log T)^{\beta}}{\Gamma(\beta+1)}+\frac{(\log T)^{\beta-1}}{|\Delta|}\left(\frac{\sum_{i=1}^{m} \mu_{i}\left(\log \xi_{i}\right)^{\beta+q}}{\Gamma(\beta+q+1)}+\frac{(\log T)^{\beta}}{\Gamma(\beta+1)}\right), \\
& N_{4}=\frac{(\log T)^{\beta-\delta}}{\Gamma(\beta-\delta+1)}+\frac{\left.\Gamma(\beta)(\log t)^{\beta-\delta-1}\right)}{\Gamma(\beta-\delta)|\Delta|}\left(\frac{\sum_{i=1}^{m} \mu_{i}\left(\log \xi_{i}\right)^{\beta+q}}{\Gamma(\beta+q+1)}+\frac{(\log T)^{\beta}}{\Gamma(\beta+1)}\right),
\end{aligned}
$$

which

$$
\Lambda=\frac{1}{(\log T)^{\alpha-1}-\frac{\Gamma(\alpha)}{\Gamma(p+\alpha)} \sum_{i=1}^{m} \lambda_{i}\left(\log \eta_{i}\right)^{p+\alpha-1}},
$$

and

$$
\Delta=\frac{1}{(\log T)^{\beta-1}-\frac{\Gamma(\beta)}{\Gamma(q+\beta)} \sum_{i=1}^{m} \mu_{i}\left(\log \xi_{i}\right)^{q+\beta-1}} .
$$

We list also the following hypotheses:

(H1) The functions $f_{1}, f_{2}:[1, T] \times \mathbb{R}^{2} \rightarrow \mathbb{R}$ are continuous.

(H2) There exists a nonnegative continuous functions $a_{i}, b_{i} \in C([1, T]), i=1,2$ such that for all $t \in[1, T]$ and $\left(x_{1}, y_{1}\right),\left(x_{2}, y_{2}\right) \in \mathbb{R}^{2}$, we have

$$
\begin{aligned}
& \left|f_{1}\left(t, x_{1}, y_{1}\right)-f_{1}\left(t, x_{2}, y_{2}\right)\right| \leq a_{1}(t)\left|x_{1}-x_{2}\right|+b_{1}(t)\left|y_{1}-y_{2}\right|, \\
& \left|f_{2}\left(t, x_{1}, y_{1}\right)-f_{2}\left(t, x_{2}, y_{2}\right)\right| \leq a_{2}(t)\left|x_{1}-x_{2}\right|+b_{2}(t)\left|y_{1}-y_{2}\right|,
\end{aligned}
$$

with

$$
\begin{gathered}
\omega_{1}=\sup _{t \in[1, T]} a_{1}(t), \omega_{2}=\sup _{t \in[1, T]} b_{1}(t), \\
\varpi_{1}=\sup _{t \in[1, T]} a_{2}(t), \varpi_{2}=\sup _{t \in[1, T]} b_{2}(t) .
\end{gathered}
$$


Existence and uniqueness results for a coupled system of Hadamard fractional... $\quad 847$

(H3) There exists a nonnegative functions $l_{1}(t)$ and $l_{2}(t)$ such that $\left|f_{1}(t, x, y)\right| \leq l_{1}(t),\left|f_{2}(t, x, y)\right| \leq l_{2}(t)$ for each $t \in[1, T]$ and all $x, y \in \mathbb{R}$, with

$$
L_{1}=\sup _{t \in[1, T]} l_{1}(t), L_{2}=\sup _{t \in[1, T]} l_{2}(t)
$$

Our first result is based on Banach contraction principle:

Theorem 3.1. Suppose that the hypothesis (H2) holds.

If

$$
\left(N_{1}+N_{2}\right)\left(\omega_{1}+\omega_{2}\right)+\left(N_{3}+N_{4}\right)\left(\varpi_{1}+\varpi_{2}\right)<1
$$

then the boundary value problem (1.1) has a unique solution on $[1, T]$.

Proof. Consider the operator $\phi: X \times Y \rightarrow X \times Y$ defined by:

$$
\phi(x, y)(t):=\left(\phi_{1} y(t), \phi_{2} x(t)\right), t \in[1, T],
$$

where

$$
\begin{aligned}
\phi_{1} y(t):= & \frac{1}{\Gamma(\alpha)} \int_{1}^{t}\left(\log \frac{t}{s}\right)^{\alpha-1} \frac{f_{1}\left(s, y(s), D^{\delta} y(s)\right)}{s} d s \\
& +\frac{(\log t)^{\alpha-1}}{\Lambda}\left[\frac{\sum_{i=1}^{m} \lambda_{i}}{\Gamma(\alpha+p)} \int_{1}^{\eta_{i}}\left(\log \frac{\eta_{i}}{s}\right)^{\alpha+p-1} \frac{f_{1}\left(s, y(s), D^{\delta} y(s)\right)}{s} d s\right. \\
& \left.-\frac{1}{\Gamma(\beta)} \int_{1}^{T}\left(\log \frac{T}{s}\right)^{\beta-1} \frac{f_{2}\left(s, x(s), D^{\sigma} x(s)\right)}{s} d s\right] .
\end{aligned}
$$

and

$$
\begin{gathered}
\phi_{2} x(t):=\frac{1}{\Gamma(\beta)} \int_{1}^{t}\left(\log \frac{t}{s}\right)^{\beta-1} \frac{f_{2}\left(s, x(s), D^{\sigma} x(s)\right)}{s} d s \\
+\frac{(\log t)^{\beta-1}}{\Delta}\left[\frac{\sum_{i=1}^{m} \mu_{i}}{\Gamma(q+\beta)} \int_{1}^{\xi_{i}}\left(\log \frac{\xi_{i}}{s}\right)^{\beta+q-1} \frac{f_{2}\left(s, x(s), D^{\sigma} x(s)\right)}{s} d s\right. \\
+\frac{(\log t)^{\beta-1}}{\Delta}\left[\frac{\sum_{i=1}^{m} \mu_{i}}{\Gamma(q+\beta)} \int_{1}^{\xi_{i}}\left(\log \frac{\xi_{i}}{s}\right)^{\beta+q-1} \frac{f_{2}\left(s, x(s), D^{\sigma} x(s)\right)}{s} d s\right.
\end{gathered}
$$

We shall prove that $\phi$ is contraction mapping.

Let $(x, y),\left(x_{1}, y_{1}\right) \in X \times Y$. Then, for each $t \in[1, T]$, we have:

$$
\begin{aligned}
\left|\phi_{1} y(t)-\phi_{1} y_{1}(t)\right| \leq & \frac{1}{\Gamma(\alpha)} \int_{1}^{t}\left(\log \frac{t}{s}\right)^{\alpha-1} \frac{\left|f_{1}\left(s, y(s), D^{\delta} y(s)\right)-f_{1}\left(s, y_{1}(s), D^{\delta} y_{1}(s)\right)\right|}{s} d s \\
& \quad+\frac{(\log T)^{\alpha-1}}{|\Lambda|}\left[\frac{\sum_{i=1}^{m} \lambda_{i}}{\Gamma(p+\alpha)} \times\right. \\
& \int_{1}^{\eta_{i}}\left(\log \frac{\eta_{i}}{s}\right)^{\alpha+p-1} \frac{\left|f_{1}\left(s, y(s), D^{\delta} y(s)\right)-f_{1}\left(s, y_{1}(s), D^{\delta} y_{1}(s)\right)\right|}{s} d s \\
+ & \left.\frac{1}{\Gamma(\alpha)} \int_{1}^{T}\left(\log \frac{T}{s}\right)^{\alpha-1} \frac{\left|f_{1}\left(s, y(s), D^{\delta} y(s)\right)-f_{1}\left(s, y_{1}(s), D^{\delta} y_{1}(s)\right)\right|}{s} d s\right] .
\end{aligned}
$$


Thanks to (H2), we obtain

$$
\begin{aligned}
& \left|\phi_{1} y(t)-\phi_{1} y_{1}(t)\right| \\
\leq & \frac{1}{\Gamma(\alpha)} \int_{1}^{t}\left(\log \frac{t}{s}\right)^{\alpha-1} \frac{\omega_{1}\left\|y-y_{1}\right\|+\omega_{2}\left\|D^{\delta} y-D^{\delta} y_{1}\right\|}{s} d s \\
+ & \left|\frac{(\log T)^{\alpha-1}}{\Lambda}\right| \frac{\sum_{i=1}^{m} \lambda_{i}}{\Gamma(p+\alpha)} \int_{1}^{\eta_{i}}\left(\log \frac{\eta_{i}}{s}\right)^{\alpha+p-1} \frac{\omega_{1}\left\|y-y_{1}\right\|+\omega_{2}\left\|D^{\delta} y-D^{\delta} y_{1}\right\|}{s} d s \\
+ & \frac{1}{\Gamma(\alpha)} \int_{1}^{T}\left(\log \frac{T}{s}\right)^{\alpha-1} \frac{\omega_{1}\left\|y-y_{1}\right\|+\omega_{2}\left\|D^{\delta} y-D^{\delta} y_{1}\right\|}{s} d s .
\end{aligned}
$$

Consequently,

$$
\begin{gathered}
\left|\phi_{1} y(t)-\phi_{1} y_{1}(t)\right| \\
\leq\left[\frac{(\log T)^{\alpha}}{\Gamma(\alpha+1)}+\frac{(\log T)^{\alpha-1}}{|\Lambda|}\left(\frac{\sum_{i=1}^{m} \lambda_{i}\left(\log \eta_{i}\right)^{p+\alpha}}{\Gamma(\alpha+p+1)}+\frac{(\log T)^{\alpha}}{\Gamma(\alpha+1)}\right)\right]\left(\omega_{1}+\omega_{2}\right) \\
\times\left(\left\|y-y_{1}\right\|+\left\|D^{\delta} y-D^{\delta} y_{1}\right\|\right)
\end{gathered}
$$

which implies that

$$
\begin{gathered}
\left\|\phi_{1}(y)-\phi_{1}\left(y_{1}\right)\right\| \leq N_{1}\left(\omega_{1}+\omega_{2}\right)\left(\left\|y-y_{1}\right\|+\left\|D^{\delta} y-D^{\delta} y_{1}\right\|\right), \\
\quad\left|D^{\sigma} \phi_{1} y(t)-D^{\sigma} \phi_{1} y_{1}(t)\right| \\
\leq \frac{1}{\Gamma(\alpha-\sigma)} \int_{1}^{t}\left(\log \frac{t}{s}\right)^{\alpha-\sigma-1} \frac{\left|f\left(s, y(s), D^{\delta} y(s)\right)-f\left(s, y_{1}(s), D^{\delta} y_{1}(s)\right)\right|}{s} d s \\
\quad+\frac{\Gamma(\alpha)(\log t)^{\alpha-\sigma-1}}{\Gamma(\alpha-\sigma)|\Lambda|} \times \\
{\left[\frac{\sum_{i=1}^{m} \lambda_{i}}{\Gamma(\alpha+p)} \int_{1}^{\eta_{i}}\left(\log \frac{\eta_{i}}{s}\right)^{\alpha+p-1} \frac{\left|f_{1}\left(s, y(s), D^{\delta} y(s)\right)-f_{1}\left(s, y_{1}(s), D^{\delta} y_{1}(s)\right)\right|}{s} d s\right.} \\
\left.+\frac{1}{\Gamma(\alpha)} \int_{1}^{T}\left(\log \frac{T}{s}\right)^{\alpha-1} \frac{\left|f_{1}\left(s, y(s), D^{\delta} y(s)\right)-f_{1}\left(s, y_{1}(s), D^{\delta} y_{1}(s)\right)\right|}{s} d s\right] .
\end{gathered}
$$

By (H2), we have

$$
\begin{aligned}
& \quad\left|D^{\sigma} \phi_{1} y(t)-D^{\sigma} \phi_{1} y_{1}(t)\right| \\
& \leq \frac{(\log T)^{\alpha-\sigma}\left(\omega_{1}+\omega_{2}\right)\left(\left\|y-y_{1}\right\|+\left\|D^{\delta} y-D^{\delta} y_{1}\right\|\right)}{\Gamma(\alpha-\sigma+1)} \\
& \quad+\frac{\Gamma(\alpha)(\log T)^{\alpha-\sigma-1}}{\Gamma(\alpha-\sigma)|\Lambda|}\left(\frac{\sum_{i=1}^{m} \lambda_{i}\left(\log \eta_{i}\right)^{\alpha+p}}{\Gamma(\alpha+p+1)}+\frac{(\log T)^{\alpha}}{\Gamma(\alpha+1)}\right)\left(\omega_{1}+\omega_{2}\right) \\
& \quad \times\left(\left\|y-y_{1}\right\|+\left\|D^{\delta} y-D^{\delta} y_{1}\right\|\right) .
\end{aligned}
$$

Hence,

$$
\begin{aligned}
& \left|D^{\sigma} \phi_{1} y(t)-D^{\sigma} \phi_{1} y_{1}(t)\right| \\
& \leq\left[\frac{(\log T)^{\alpha-\sigma}}{\Gamma(\alpha-\sigma+1)}+\frac{\Gamma(\alpha)(\log T)^{\alpha-\sigma-1}}{\Gamma(\alpha-\sigma)|\Lambda|}\left(\frac{\sum_{i=1}^{m} \lambda_{i}\left(\log \eta_{i}\right)^{\alpha+p}}{\Gamma(\alpha+p+1)}+\frac{(\log T)^{\alpha}}{\Gamma(\alpha+1)}\right)\right] \times \\
& \quad\left(\omega_{1}+\omega_{2}\right)\left(\left\|y-y_{1}\right\|+\left\|D^{\delta} y-D^{\delta} y_{1}\right\|\right) .
\end{aligned}
$$

Therefore,

$$
\left|D^{\sigma} \phi_{1} y(t)-D^{\sigma} \phi_{1} y_{1}(t)\right| \leq N_{2}\left(\omega_{1}+\omega_{2}\right)\left(\left\|y-y_{1}\right\|+\left\|D^{\delta} y-D^{\delta} y_{1}\right\|\right) .
$$

Consequently,

$$
\left\|D^{\sigma} \phi_{1}(y)-D^{\sigma} \phi_{1}\left(y_{1}\right)\right\| \leq N_{2}\left(\omega_{1}+\omega_{2}\right)\left(\left\|y-y_{1}\right\|+\left\|D^{\delta} y-D^{\delta} y_{1}\right\|\right) .
$$


By (??) and (3.11), we can write

$$
\left\|\phi_{1}(y)-\phi_{1}\left(y_{1}\right)\right\|_{X} \leq\left(N_{1}+N_{2}\right)\left(\omega_{1}+\omega_{2}\right)\left(\left\|y-y_{1}\right\|+\left\|D^{\delta} y-D^{\delta} y_{1}\right\|\right) .
$$

With the same arguments as before, we have

$$
\left\|\phi_{2}(x)-\phi_{2}\left(x_{1}\right)\right\|_{Y} \leq\left(N_{3}+N_{4}\right)\left(\varpi_{1}+\varpi_{2}\right)\left(\left\|x-x_{1}\right\|+\left\|D^{\sigma} x-D^{\sigma} x_{1}\right\|\right) .
$$

And by,(3.12) and (3.13), we obtain

$$
\begin{aligned}
& \left\|\phi(x, y)-\phi\left(x_{1}, y_{1}\right)\right\|_{X \times Y} \\
\leq & {\left[\left(N_{1}+N_{2}\right)\left(\omega_{1}+\omega_{2}\right)+\left(N_{3}+N_{4}\right)\left(\varpi_{1}+\varpi_{2}\right)\right]\left\|\left(x-x_{1}, y-y_{1}\right)\right\|_{X \times Y} . }
\end{aligned}
$$

Thanks to (3.1), we conclude that $\phi$ is contraction. As a consequence of Banach fixed point theorem, we deduce that $\phi$ has a fixed point which is a solution of the coupled system (1.1).

The second main result is the following theorem:

Theorem 3.2. Assume that the hypotheses (H1) and (H3) are satisfied.

Then, the coupled system (1.1) has at least a solution on $[1, T]$.

Proof. We shall use Scheafer's fixed point theorem to prove that $\phi$ has at least a fixed point on $X \times Y$. It is to note that $\phi$ is continuous on $X \times Y$ in view of the continuity of $f_{1}$ and $f_{2}$ (hypothesis (H1)).

Now, We shall prove that $\phi$ maps bounded sets into bounded sets in $X \times Y$ : Taking $r>0$, and $(x, y) \in B_{r}, B_{r}:=\left\{(x, y) \in X \times Y ;\|(x, y)\|_{X \times Y} \leq r\right\}$, then for each $t \in[1, T]$, we have:

$$
\begin{aligned}
\left|\phi_{1} y(t)\right| & \leq \frac{1}{\Gamma(\alpha)} \int_{1}^{t}\left(\log \frac{t}{s}\right)^{\alpha-1} \frac{\left|f_{1}\left(s, y(s), D^{\delta} y(s)\right)\right|}{s} d s \\
& +\frac{(\log t)^{\alpha-1}}{|\Lambda|}\left[\frac{\sum_{i=1}^{m} \lambda_{i}}{\Gamma(\alpha+p)} \int_{1}^{\eta_{i}}\left(\log \frac{\eta_{i}}{s}\right)^{\alpha+p-1} \frac{\left|f_{1}\left(s, y(s), D^{\delta} y(s)\right)\right|}{s} d s\right. \\
& \left.+\frac{1}{\Gamma(\alpha)} \int_{1}^{T}\left(\log \frac{T}{s}\right)^{\alpha-1} \frac{\left|f_{1}\left(s, y(s), D^{\delta} y(s)\right)\right|}{s} d s\right] .
\end{aligned}
$$

Thanks to (H3), we can write

$$
\begin{aligned}
\left|\phi_{1} y(t)\right| & \leq \frac{1}{\Gamma(\alpha)} \int_{1}^{t}\left(\log \frac{t}{s}\right)^{\alpha-1} \frac{\sup _{t \in J} l_{1}(t)}{s} d s \\
& +\frac{(\log t)^{\alpha-1}}{|\Lambda|}\left[\frac{\sum_{i=1}^{m} \lambda_{i}}{\Gamma(\alpha+p)} \int_{1}^{\eta_{i}}\left(\log \frac{\eta_{i}}{s}\right)^{\alpha+p-1} \frac{\sup _{t \in J} l_{1}(t)}{s} d s\right. \\
& \left.+\frac{1}{\Gamma(\alpha)} \int_{1}^{T}\left(\log \frac{T}{s}\right)^{\alpha-1} \frac{\sup _{t \in J} l_{1}(t)}{s} d s\right] . \\
& \leq \sup _{t \in J} l_{1}(t)\left[\frac{(\log T)^{\alpha}}{\Gamma(\alpha+1)}+\frac{(\log T)^{\alpha-1}}{|\Lambda|}\left(\frac{\sum_{i=1}^{m} \lambda_{i}\left(\log \eta_{i}\right)^{\alpha+p}}{\Gamma(\alpha+p+1)}+\frac{(\log T)^{\alpha}}{\Gamma(\alpha+1)}\right)\right] .
\end{aligned}
$$


Therefore,

$$
\begin{aligned}
& \left|\phi_{1} y(t)\right| \\
\leq & L_{1}\left[\frac{(\log T)^{\alpha}}{\Gamma(\alpha+1)}+\frac{(\log T)^{\alpha-1}}{|\Lambda|}\left(\frac{\sum_{i=1}^{m} \lambda_{i}\left(\log \eta_{i}\right)^{\alpha+p}}{\Gamma(\alpha+p+1)}+\frac{(\log T)^{\alpha}}{\Gamma(\alpha+1)}\right)\right], t \in[1, T] .
\end{aligned}
$$

Hence, we have

$$
\begin{aligned}
& \left\|\phi_{1}(y)\right\| \\
\leq & L_{1}\left[\frac{(\log T)^{\alpha}}{\Gamma(\alpha+1)}+\frac{(\log T)^{\alpha-1}}{|\Lambda|}\left(\frac{\sum_{i=1}^{m} \lambda_{i}\left(\log \eta_{i}\right)^{\alpha+p}}{\Gamma(\alpha+p+1)}+\frac{(\log T)^{\alpha}}{\Gamma(\alpha+1)}\right)\right]=L_{1} N_{1} .
\end{aligned}
$$

On the other hand,

$$
\begin{aligned}
\left|D^{\sigma} \phi_{1} y(t)\right| \leq & \frac{1}{\Gamma(\alpha-\sigma)} \int_{1}^{t}\left(\log \frac{t}{s}\right)^{\alpha-\sigma-1} \frac{\left|f\left(s, y(s), D^{\delta} y(s)\right)\right|}{s} d s \\
& +\frac{\Gamma(\alpha)(\log t)^{\alpha-\sigma-1}}{\Gamma(\alpha-\sigma)|\Lambda|}\left[\frac{\sum_{i=1}^{m} \lambda_{i}}{\Gamma(\alpha+p)} \int_{1}^{\eta_{i}}\left(\log \frac{\eta_{i}}{s}\right)^{\alpha+p-1} \frac{\left|f_{1}\left(s, y(s), D^{\delta} y(s)\right)\right|}{s} d s\right. \\
& \left.+\frac{1}{\Gamma(\alpha)} \int_{1}^{T}\left(\log \frac{T}{s}\right)^{\alpha-1} \frac{\left|f_{1}\left(s, y(s), D^{\delta} y(s)\right)\right|}{s} d s\right] .
\end{aligned}
$$

By (H3), we have,

$$
\begin{gathered}
\left|D^{\sigma} \phi_{1} y(t)\right| \\
\leq L_{1}\left[\frac{(\log T)^{\alpha-\sigma}}{\Gamma(\alpha-\sigma+1)}+\frac{\Gamma(\alpha)(\log T)^{\alpha-\sigma-1}}{\Gamma(\alpha-\sigma)|\Lambda|}\left(\frac{\sum_{i=1}^{m} \lambda_{i}\left(\log \eta_{i}\right)^{\alpha+p}}{\Gamma(\alpha+p+1)}+\frac{(\log T)^{\alpha}}{\Gamma(\alpha+1)}\right)\right] .
\end{gathered}
$$

Consequently we obtain,

$$
\left|D^{\sigma} \phi_{1} y(t)\right| \leq L_{1} N_{2}, t \in[1, T] .
$$

Therefore,

$$
\left\|D^{\sigma} \phi_{1}(y)\right\| \leq L_{1} N_{2} .
$$

Combining (3.18) and (3.21), yields

$$
\left\|\phi_{1}(y)\right\|_{X} \leq L_{1}\left(N_{1}+N_{2}\right) .
$$

Similarly, it can be shown that,

$$
\left\|\phi_{2}(x)\right\|_{Y} \leq L_{2}\left(N_{3}+N_{4}\right) .
$$

It follows from (3.22) and (3.23) that

$$
\|\phi(x, y)\|_{X \times Y} \leq L_{1}\left(N_{1}+N_{2}\right)+L_{2}\left(N_{3}+N_{4}\right) .
$$

Consequently

$$
\|\phi(x, y)\|_{X \times Y}<\infty .
$$


Next, we will prove that $\phi$ is equicontinuous on $[1, T]$ : For $(x, y) \in B_{r}$, and $t_{1}, t_{2} \in$ $[1, T]$, such that $t_{1}<t_{2}$. Thanks hypothesis (H3), we have:

$$
\begin{aligned}
\left|\phi_{1} y\left(t_{2}\right)-\phi_{1} y\left(t_{1}\right)\right| \leq & \frac{L_{1}}{\Gamma(\alpha)}\left|\int_{1}^{t_{1}}\left(\left(\log \frac{t_{1}}{s}\right)^{\alpha-1}-\left(\log \frac{t_{2}}{s}\right)^{\alpha-1}\right) \frac{1}{s} d s\right| \\
+ & \frac{L_{1}}{\Gamma(\alpha)}\left|\int_{t_{1}}^{t_{2}}\left(\log \frac{t_{2}}{s}\right)^{\alpha-1} \frac{1}{s} d s\right| \\
& +\frac{L_{1}}{\Gamma(\alpha)} \frac{\left(\log t_{1}\right)^{\alpha-1}-\left(\log t_{2}\right)^{\alpha-1}}{\Lambda} \frac{\sum_{i=1}^{m} \lambda_{i}}{\Gamma(\alpha+p)} \int_{1}^{\eta_{i}}\left(\log \frac{\eta_{i}}{s}\right)^{\alpha+p-1} \frac{1}{s} d s \\
& -\frac{1}{\Gamma(\alpha)} \int_{1}^{T}\left(\log \frac{T}{s}\right)^{\alpha-1} \frac{1}{s} d s .
\end{aligned}
$$

Thus,

$$
\begin{aligned}
\left|\phi_{1} y\left(t_{2}\right)-\phi_{1} y\left(t_{1}\right)\right| & \leq \frac{L_{1}}{\Gamma(\alpha)}\left|\int_{1}^{t_{1}}\left(\left(\log \frac{t_{1}}{s}\right)^{\alpha-1}-\left(\log \frac{t_{2}}{s}\right)^{\alpha-1}\right) \frac{1}{s} d s\right| \\
& +\frac{L_{1}}{\Gamma(\alpha)}\left|\int_{t_{1}}^{t_{2}}\left(\log \frac{t_{2}}{s}\right)^{\alpha-1} \frac{1}{s} d s\right| \\
+ & L_{1}\left|\frac{\left(\log t_{1}\right)^{\alpha-1}-\left(\log t_{2}\right)^{\alpha-1}}{\Lambda}\right|\left(\frac{\sum_{i=1}^{m} \lambda_{i}\left(\log \eta_{i}\right)^{\alpha+p}}{\Gamma(\alpha+p+1)}+\frac{(\log T)^{\alpha}}{\Gamma(\alpha+1)}\right) .
\end{aligned}
$$

and using (H3), we obtain:

$$
\begin{aligned}
& \left|D^{\sigma} \phi_{1} y\left(t_{2}\right)-D^{\sigma} \phi_{1} y\left(t_{1}\right)\right| \\
& \leq \frac{L_{1}}{\Gamma(\alpha-\sigma)}\left|\int_{1}^{t_{1}}\left(\left(\log \frac{t_{1}}{s}\right)^{\alpha-\sigma-1}-\left(\log \frac{t_{2}}{s}\right)^{\alpha-\sigma-1}\right) \frac{1}{s} d s\right| \\
& +\frac{L_{1}}{\Gamma(\alpha-\sigma)}\left|\int_{t_{1}}^{t_{2}}\left(\log \frac{t_{2}}{s}\right)^{\alpha-\sigma-1} \frac{1}{s} d s\right|
\end{aligned}
$$

Hence, by (3.26) and (3.27), we can write

$$
\begin{gathered}
\left\|\phi_{1} y\left(t_{2}\right)-\phi_{1} y\left(t_{1}\right)\right\|_{X} \leq \frac{L_{1}}{\Gamma(\alpha)}\left|\int_{1}^{t_{1}}\left(\left(\log \frac{t_{1}}{s}\right)^{\alpha-1}-\left(\log \frac{t_{2}}{s}\right)^{\alpha-1}\right) \frac{1}{s} d s\right| \\
+\frac{L_{1}}{\Gamma(\alpha)}\left|\int_{t_{1}}^{t_{2}}\left(\log \frac{t_{2}}{s}\right)^{\alpha-1} \frac{1}{s} d s\right| \\
+L_{1}\left|\frac{\left(\log t_{1}\right)^{\alpha-1}-\left(\log t_{2}\right)^{\alpha-1}}{\Lambda}\right|\left(\frac{\sum_{i=1}^{m} \lambda_{i}\left(\log \eta_{i}\right)^{\alpha+p}}{\Gamma(\alpha+p+1)}+\frac{(\log T)^{\alpha}}{\Gamma(\alpha+1)}\right) \\
+\frac{L_{1}}{\Gamma(\alpha-\sigma)}\left|\int_{1}^{t_{1}}\left(\left(\log \frac{t_{1}}{s}\right)^{\alpha-\sigma-1}-\left(\log \frac{t_{2}}{s}\right)^{\alpha-\sigma-1}\right) \frac{1}{s} d s\right| \\
+\frac{L_{1}}{\Gamma(\alpha-\sigma)}\left|\int_{t_{1}}^{t_{2}}\left(\log \frac{t_{2}}{s}\right)^{\alpha-\sigma-1} \frac{1}{s} d s\right| \\
+\frac{L_{1} \Gamma(\alpha)}{\Gamma(\alpha-\sigma)}\left|\frac{\left(\log t_{1}\right)^{\alpha-\sigma-1}-\left(\log t_{2}\right)^{\alpha-\sigma-1}}{\Lambda}\right|\left(\frac{\sum_{i=1}^{m} \lambda_{i}\left(\log \eta_{i}\right)^{\alpha+p}}{\Gamma(\alpha+p+1)}+\frac{(\log T)^{\alpha}}{\Gamma(\alpha+1)}\right) .
\end{gathered}
$$

With the same arguments as before, we get

$$
\begin{aligned}
\| \phi_{1} x( & \left.t_{2}\right)-\phi_{1} x\left(t_{1}\right) \|_{Y} \\
& \leq \frac{L_{2}}{\Gamma(\beta)}\left|\int_{1}^{t_{1}}\left(\left(\log \frac{t_{1}}{s}\right)^{\beta-1}-\left(\log \frac{t_{2}}{s}\right)^{\beta-1}\right) \frac{1}{s} d s\right| \\
& +\frac{L_{2}}{\Gamma(\beta)}\left|\int_{t_{1}}^{t_{2}}\left(\log \frac{t_{2}}{s}\right)^{\beta-1} \frac{1}{s} d s\right| \\
& +L_{2}\left|\frac{\left(\log t_{1}\right)^{\beta-1}-\left(\log t_{2}\right)^{\beta-1}}{\Delta}\right|\left(\frac{\sum_{i=1}^{m} \mu_{i}\left(\log \xi_{i}\right)^{\beta+q}}{\Gamma(\beta+q+1)}+\frac{(\log T)^{\beta}}{\Gamma(\beta+1)}\right) \\
& +\frac{L_{2}}{\Gamma(\beta-\delta)}\left|\int_{1}^{t_{1}}\left(\left(\log \frac{t_{1}}{s}\right)^{\beta-\delta-1}-\left(\log \frac{t_{2}}{s}\right)^{\beta-\delta-1}\right) \frac{1}{s} d s\right| \\
& +\frac{L_{2}}{\Gamma(\beta-\delta)}\left|\int_{t_{1}}^{t_{2}}\left(\log \frac{t_{2}}{s}\right)^{\beta-\delta-1} \frac{1}{s} d s\right| \\
& +\frac{L_{2} \Gamma(\beta)}{\Gamma(\beta-\delta)}\left|\frac{\left(\log t_{1}\right)^{\beta-\delta-1}-\left(\log t_{2}\right)^{\beta-\delta-1}}{\Delta}\right|\left(\frac{\sum_{i=1}^{m} \mu_{i}\left(\log \xi_{i}\right)^{\beta+q}}{\Gamma(\beta+q+1)}+\frac{(\log T)^{\beta}}{\Gamma(\beta+1)}\right) .
\end{aligned}
$$


Thanks to (3.28) and (3.29), we can state that $\left\|\phi(x, y)\left(t_{2}\right)-\phi(x, y)\left(t_{1}\right)\right\|_{X \times Y} \rightarrow$ 0 as $t_{2} \rightarrow t_{1}$ and by Arzela-Ascoli theorem, we conclude that $\phi$ is completely continuous operator.

Finally, we shall show that the set $\Omega$ defined by

$$
\Omega=\{(x, y) \in X \times Y,(x, y)=\rho \phi(x, y), 0<\rho<1\},
$$

is bounded:

Let $(x, y) \in \Omega$, then $(x, y)=\rho \phi(x, y)$, for some $0<\rho<1$. Thus, for each $t \in[1, T]$, we have:

$$
x(t)=\rho \phi_{1} y(t), y(t)=\rho \phi_{2} x(t) .
$$

Then

$$
\begin{aligned}
\frac{1}{\rho}|x(t)| & \leq \frac{1}{\Gamma(\alpha)} \int_{1}^{t}\left(\log \frac{t}{s}\right)^{\alpha-1} \frac{\left|f_{1}\left(s, y(s), D^{\delta} y(s)\right)\right|}{s} d s \\
& +\frac{\left|(\log T)^{\alpha-1}\right|}{|\Lambda|}\left[\frac{\sum_{i=1}^{m} \lambda_{i}}{\Gamma(\alpha+p)} \int_{1}^{\eta_{i}}\left(\log \frac{\eta_{i}}{s}\right)^{\alpha+p-1} \frac{\left|f_{1}\left(s, y(s), D^{\delta} y(s)\right)\right|}{s} d s\right. \\
& \left.+\frac{1}{\Gamma(\alpha)} \int_{1}^{T}\left(\log \frac{T}{s}\right)^{\alpha-1} \frac{\left|f_{1}\left(s, y(s), D^{\delta} y(s)\right)\right|}{s} d s\right] .
\end{aligned}
$$

Thanks to (H3), we can write

$$
\begin{aligned}
\frac{1}{\rho}|x(t)| & \leq \frac{L_{1}}{\Gamma(\alpha)} \int_{1}^{t}\left(\log \frac{t}{s}\right)^{\alpha-1} \frac{1}{s} d s \\
& +\frac{L_{1}(\log T)^{\alpha-1}}{|\Lambda|}\left[\frac{\sum_{i=1}^{m} \lambda_{i}}{\Gamma(\alpha+p)} \int_{1}^{\eta_{i}}\left(\log \frac{\eta_{i}}{s}\right)^{\alpha+p-1} \frac{1}{s} d s\right. \\
& \left.+\frac{1}{\Gamma(\alpha)} \int_{1}^{T}\left(\log \frac{T}{s}\right)^{\alpha-1} \frac{1}{s} d s\right] .
\end{aligned}
$$

Therefore,

$$
|x(t)| \leq \rho L_{1}\left[\frac{(\log T)^{\alpha}}{\Gamma(\alpha+1)}+\frac{(\log T)^{\alpha-1}}{|\Lambda|}\left(\frac{\sum_{i=1}^{m} \lambda_{i}\left(\log \eta_{i}\right)^{\alpha+p}}{\Gamma(\alpha+p+1)}+\frac{(\log T)^{\alpha}}{\Gamma(\alpha+1)}\right)\right]
$$

Hence,

$$
|x(t)| \leq \rho L_{1} N_{1} .
$$

On the other hand,

$$
\begin{aligned}
& \frac{1}{\rho}\left|D^{\sigma} x(t)\right| \\
& \leq \frac{1}{\Gamma(\alpha-\sigma)} \int_{1}^{t}\left(\log \frac{t}{s}\right)^{\alpha-\sigma-1} \frac{\left|f\left(s, y(s), D^{\delta} y(s)\right)\right|}{s} d s \\
& +\frac{\Gamma(\alpha)(\log t)^{\alpha-\sigma-1}}{\Gamma(\alpha-\sigma)|\Lambda|}\left[\frac{\sum_{i=1}^{m} \lambda_{i}}{\Gamma(\alpha+p)} \int_{1}^{\eta_{i}}\left(\log \frac{\eta_{i}}{s}\right)^{\alpha+p-1} \frac{\left|f_{1}\left(s, y(s), D^{\delta} y(s)\right)\right|}{s} d s\right. \\
& \left.+\frac{1}{\Gamma(\alpha)} \int_{1}^{T}\left(\log \frac{T}{s}\right)^{\alpha-1} \frac{\left|f_{1}\left(s, y(s), D^{\delta} y(s)\right)\right|}{s} d s\right] .
\end{aligned}
$$


By (H3), we have

$$
\begin{aligned}
& \frac{1}{\rho}\left|D^{\sigma} x(t)\right| \\
\leq & L_{1}\left[\frac{(\log T)^{\alpha-\sigma}}{\Gamma(\alpha-\sigma+1)}+\frac{\Gamma(\alpha)(\log T)^{\alpha-\sigma-1}}{\Gamma(\alpha-\sigma)|\Lambda|}\left(\frac{\sum_{i=1}^{m} \lambda_{i}\left(\log \eta_{i}\right)^{\alpha+p}}{\Gamma(\alpha+p+1)}+\frac{(\log T)^{\alpha}}{\Gamma(\alpha+1)}\right)\right] .
\end{aligned}
$$

Therefore,

$$
\begin{aligned}
& \left|D^{\sigma} x(t)\right| \\
\leq & \rho L_{1}\left[\frac{(\log T)^{\alpha-\sigma}}{\Gamma(\alpha-\sigma+1)}+\frac{\Gamma(\alpha)(\log T)^{\alpha-\sigma-1}}{\Gamma(\alpha-\sigma)|\Lambda|}\left(\frac{\sum_{i=1}^{m} \lambda_{i}\left(\log \eta_{i}\right)^{\alpha+p}}{\Gamma(\alpha+p+1)}+\frac{(\log T)^{\alpha}}{\Gamma(\alpha+1)}\right)\right] .
\end{aligned}
$$

Thus,

$$
\left\|D^{\sigma}(x)\right\| \leq \rho L_{1} N_{2} .
$$

From (3.35) and (3.39) we get

$$
\|x\|_{X} \leq \rho L_{1}\left(N_{1}+N_{2}\right) .
$$

Analogously, we can obtain

$$
\|y\|_{Y} \leq \rho L_{2}\left(N_{3}+N_{4}\right) .
$$

It follows from (3.40) and (3.41) that

$$
\|(x, y)\|_{X \times Y} \leq \rho\left[L_{1}\left(N_{1}+N_{2}\right)+L_{2}\left(N_{3}+N_{4}\right)\right] .
$$

Hence,

$$
\|\phi(x, y)\|_{X \times Y}<\infty .
$$

This shows that the set $\Omega$ is bounded.

As a consequence of Schaefer's fixed point theorem, we deduce that $\phi$ has at least a fixed point, which is a solution of coupled system (1.1).

\section{Examples}

Example 4.1. Let us consider the Hadamard coupled system:

$$
\left\{\begin{array}{l}
D^{\frac{5}{3}} x(t)=\frac{1}{8(t+2)^{2}}\left(\frac{|y(t)|}{1+|y(t)|}+\frac{t\left|D^{\frac{1}{2}} y(t)\right|}{2 \pi\left(1+\left|D^{\frac{1}{2}} y(t)\right|\right)}\right)+\cos \left(1+t+t^{2}\right), t \in[1, e], \\
D^{\frac{5}{3}} y(t)=\frac{1}{20 \pi+t^{2}}\left(\sin |x(t)|+\frac{t+t^{2}}{\pi} \sin \left|D^{\frac{1}{3}} x(t)\right|\right)+\ln \left(2+t^{2}\right), t \in[1, e], \\
x(1)=0, x(e)=2 I^{\frac{3}{2}} x\left(\frac{5}{4}\right)+I^{\frac{3}{2}} x\left(\frac{4}{3}\right)+\frac{7}{4} I^{\frac{3}{2}} x\left(\frac{7}{5}\right), \\
y(1)=0, y(e)=\frac{3}{2} I^{\frac{4}{3}} x\left(\frac{7}{6}\right)+\frac{6}{5} I^{\frac{4}{3}} x\left(\frac{5}{4}\right)+\frac{7}{6} I^{\frac{4}{3}} x\left(\frac{3}{2}\right) .
\end{array}\right.
$$

For this example, we have for $t \in[1, e]$

$$
\begin{gathered}
f_{1}(t, x, y)=\frac{1}{8(t+2)^{2}}\left(\frac{|x|}{1+|x|}+\frac{t|y|}{2 \pi(1+|y|)}\right)+\cos \left(1+t+t^{2}\right), x, y \in \mathbb{R} \\
f_{2}(t, x, y)=\frac{1}{20 \pi+t^{2}}\left(\sin |x|+\frac{t+t^{2}}{\pi} \sin |y|\right)+\ln \left(2+t^{2}\right), x, y \in \mathbb{R} .
\end{gathered}
$$


Taking $x, y, x_{1}, y_{1} \in \mathbb{R}, t \in[1, e]$, then:

$$
\begin{aligned}
& \left|f_{1}(t, x, y)-f_{1}\left(t, x_{1}, y_{1}\right)\right| \leq \frac{1}{8(t+2)^{2}}\left|x-x_{1}\right|+\frac{t}{16 \pi(t+2)^{2}}\left|y-y_{1}\right|, \\
& \left|f_{2}(t, x, y)-f_{2}\left(t, x_{1}, y_{1}\right)\right| \leq \frac{1}{20 \pi+t^{2}}\left|x-x_{1}\right|+\frac{t+t^{2}}{\pi\left(20 \pi+t^{2}\right)}\left|y-y_{1}\right| .
\end{aligned}
$$

So, we can take

$$
a_{1}(t)=\frac{1}{8(t+2)^{2}}, b_{1}(t)=\frac{t}{16 \pi(t+2)^{2}},
$$

and

$$
a_{2}(t)=\frac{1}{20 \pi+t^{2}}, b_{2}(t)=\frac{t+t^{2}}{\pi\left(20 \pi+t^{2}\right)} .
$$

It follows then that

$$
\begin{gathered}
\omega_{1}=\sup _{t \in[1, e]} a_{1}(t)=\frac{1}{72}, \omega_{2}=\sup _{t \in[1, e]} b_{1}(t)=\frac{1}{144 \pi}, \\
\varpi_{1}=\sup _{t \in[1, e]} a_{2}(t)=\frac{1}{20 \pi+1}, \varpi_{2}=\sup _{t \in[1, e]} b_{2}(t)=\frac{e+e^{2}}{\pi(20 \pi+e)}, \\
N_{1}=1,3234, N_{2}=1,5028, N_{3}=1,3153, N_{4}=1,4974 .
\end{gathered}
$$

and $\Delta=1.0246, \Lambda=1.020$,

$$
\left(N_{1}+N_{2}\right)\left(\omega_{1}+\omega_{2}\right)+\left(N_{3}+N_{4}\right)\left(\varpi_{1}+\varpi_{2}\right)=0,3054<1 .
$$

Hence by Theorem 5 , then the system (4.1) has a unique solution on $[1, e]$.

Example 4.2. Consider the following coupled system:

$$
\left\{\begin{array}{l}
D^{\frac{7}{4}} x(t)=\frac{\sin \left(|y(t)|+\left|D^{\frac{3}{4}} y(t)\right|\right)}{t^{2}+5 t+2}, t \in[1, e] \\
D^{\frac{6}{5}} y(t)=\frac{\cos \left(|x(t)|+\left|D^{\frac{1}{5}} x(t)\right|\right)}{t^{2}+t+20 \pi}, t \in[1, e] \\
x(1)=0, x(e)=\frac{6}{5} I^{\frac{5}{3}}\left(\frac{5}{4}\right)+\frac{7}{4} I^{\frac{5}{3}}\left(\frac{4}{3}\right)+\frac{7}{4} I^{\frac{5}{3}}\left(\frac{7}{5}\right), \\
y(1)=0, y(e)=\frac{3}{2} I^{\frac{7}{5}}\left(\frac{7}{6}\right)+\frac{6}{5} I^{\frac{7}{5}}\left(\frac{5}{4}\right)+\frac{7}{6} I^{\frac{7}{5}}\left(\frac{3}{2}\right) .
\end{array}\right.
$$

Then, we have

$$
\begin{aligned}
& f_{1}(t, x, y)=\frac{\sin \left(|y(t)|+\left|D^{\frac{3}{4}} y(t)\right|\right)}{t^{2}+5 t+2}, x, y \in \mathbb{R}, \\
& f_{2}(t, x, y)=\frac{\cos \left(|x(t)|+\left|D^{\frac{1}{5}} x(t)\right|\right)}{t^{2}+t+20 \pi}, x, y \in \mathbb{R} .
\end{aligned}
$$

Let $x, y \in \mathbb{R}$ and $t \in[1, e]$. Then

$$
\left|f_{1}(t, x, y)\right| \leq \frac{1}{t^{2}+5 t+2},\left|f_{2}(t, x, y)\right| \leq \frac{1}{t^{2}+t+20 \pi}
$$


So we take

$$
l_{1}(t)=\frac{1}{t^{2}+5 t+2}, l_{2}(t)=\frac{1}{t^{2}+t+20 \pi} .
$$

Then

$$
L_{1}=0,1250, L_{2}=0,0154 \text {. }
$$

Thanks to Theorem 6 , the system (4.2) has at least one solution on $[1, e]$.

\section{RE F E R E N C E S}

1. M. R. Ali, A.R. Hadhoud and H.M. Srivastava: Solution of fractional Volterra-Fredholm integro-differential equations under mixed boundary conditions by using the $H O B W$ method. Advances in Difference Equations. Article ID 115, (2019), 1-14.

2. B. Ahmad, S.K. Ntouyas: A fully Hadamard type integral boundary value problem of a coupled system of fractional differential equations. Fract. Calc. Appl. Anal. 17(2), (2014), 348-360.

3. A. Anber, S. Belarbi and Z. Dahmani: New existence and uniqueness results for fractional differential equations. Analele Stiintifice ale Universitatii Ovidius Constanta Seria Matematica. Vol. 21(3), (2013), 33-41.

4. C.Z. Bai and J.X. Fang: The existence of a positive solution for a singular coupled system of nonlinear fractional differential equations. Applied Mathematics and Computation. 150(3), (2004) 611-621.

5. D. Delbosco, L. Rodino: Existence and uniqueness for a nonlinear fractional differential equation. J. Math. Anal. Appl. 204, 3(4), (1996), 429-440.

6. K. Diethelm, N.J. Ford: Analysis of fractional differential equations. J. Math. Anal. Appl. 265(2), (2002), 229-248 .

7. K. Diethelm, G. Walz: Numerical solution of fraction order differential equations by extrapolation. Numer. Algorithms. 16(3), (1998), 231-253,

8. M. Houas, Z. Dahmani, M. Benbachir: New results for a boundary value problem for differential equations of arbitrary order. International Journal of Modern Mathematical Sciences. 7(2), (2013), 195-211.

9. M. Houas, Z. Dahmani: New results for a coupled system of fractional differential equations. Facta Universitatis (NIS) Ser. Math. Inform. 28(2),(2013), 133-150.

10. M. Houas, Z. Dahmani: New results for a system of two fractional differential equations involving n Caputo derivatives. Kragujevac J. Math. 38 (2014), 283-301.

11. A.M.A. El-Sayed: Nonlinear functional differential equations of arbitrary orders. Nonlinear Anal. 33(2), (1998), 181-186.

12. V. Gafiychuk, B. Datsko, and V. Meleshko: Mathematical modeling of time fractional reaction-diffusion systems. Journal of Computational and Applied Mathematics. 220(12), ( 2008), 215-225.

13. A.A. Kilbas, H.M. Srivastava, J.J. Trujillo: Theory and applications of fractional differential equations. North-Holland Mathematics Studies, 204, Elsevier Science B.V. Amsterdam, 2006. 
14. V. Lakshmikantham, A.S. Vatsala: Basic theory of fractional differential equations. Nonlinear Anal. 69(8), (2008), 2677-2682.

15. J. Liang,Z. Liu, X. Wang: Solvability for a couple system of nonlinear fractional differential equations in a Banach space. Fractional Calculus and Applied Analysis. 16(1), (2013), 51-63.

16. F. Mainardi, Fractional calculus: Some basic problem in continuum and statistical mechanics. Fractals and fractional calculus in continuum mechanics. Springer, Vienna. 1997.

17. S.K. Ntouyas: Existence results for first order boundary value problems for fractional differential equations and inclusions with fractional integral boundary conditions. Journal of Fractional Calculus and Applications. 3(9),(2012), 1-14.

18. I. Podlubny, I. Petras, B.M. Vinagre, P. O'leary, L. Dorcak: Analogue realizations of fractional-order controllers. Fractional order calculus and its applications. Nonlinear Dynam. 29(4),(2002), 281-296.

19. M.U. Rehman ,R.A Khan and N.A Asif :Three point boundary value problems for nonlinear fractional differential equations. Acta Mathematica Scientia 2011,31B(4), 1337-1346.

20. H. M. Srivastava, A.M. A. El-Sayed and F. M. Gaafar: A class of nonlinear boundary value problems for an arbitrary fractional-order differential equation with the RiemannStieltjes functional integral and infinite-point boundary conditions. Symmetry. 10(10), (2018), 1-13.

21. X. Su: Boundary value problem for a coupled system of nonlinear fractional differential equations. Applied Mathematics Letters. 22(1),(2009, 64-69 .

22. I. Tejado, B. M. Vinagre, O.E. Traver, J. Prieto-Arranz and C. Nuevo-Gallardo: Back to basics: Meaning of the parameters of fractional order PID controllers. Mathematics.7(2019), Article ID 533, 1-10.

23. J. Wang, H. Xiang, Z. Liu: Positive solution to nonzero boundary values problem for a coupled system of nonlinear fractional differential equations. International Journal of Differential Equations. Article ID 186928, 12 pages, 2010.

24. W. Yang: Positive solutions for a coupled system of nonlinear fractional differential equations with integral boundary conditions. Comput. Math. Appl. 63,(2012), 288-297.

25. Y. Zhang, Z. Bai, T. Feng: Existence results for a coupled system of nonlinear fractional three-point boundary value problems at resonance. Comput. Math. Appl. 61,(2011) 1032-1047.

\author{
Mohamed HOUAS \\ Laboratory FIMA, UDBKM, University of Khemis Miliana, Algeria \\ houasmed@yahoo.fr
}

Khellaf OULD MELHA

Department of Mathematics

University of Chlef, Algeria

k.ouldmelha@univ-chlef.dz 